\title{
Identifying prostate carcinoma by MALDI-Imaging
}

\author{
KRISTINA SCHWAMBORN ${ }^{1 *}$, RENÉ C. KRIEG $^{1 *}$, MARCUS RESKA $^{1}$, \\ GERHARD JAKSE ${ }^{2}$, RUTH KNUECHEL ${ }^{1}$ and AXEL WELLMANN ${ }^{1}$ \\ ${ }^{1}$ Institute of Pathology, ${ }^{2}$ Department of Urology, RWTH Aachen University, \\ Pauwelsstr. 30, 52074 Aachen, Germany
}

Received March 23, 2007; Accepted April 30, 2007

\begin{abstract}
Prostate cancer has become one of the most common malignancies worldwide. Although lacking in specificity its diagnosis is still based partially on the serumbased test for prostate-specific antigen. As its pathogenesis has not yet been deciphered, the ongoing search for new and more reliable biomarkers remains a challenge to stratify disease onset and progression. Matrix-assisted laser desorption/ ionization (MALDI)-Imaging is a promising technique to assist in this endeavor. It delivers accurate mass spectrometric information of the sample's proteins and enables the visualization of the spatial distribution of protein expression profiles and correlation of the information with the histomorphological features of the same tissue section. This study describes the analysis of 22 prostate sections (11 with and 11 without prostate cancer) by MALDI-Imaging. Specific protein expression patterns were obtained for normal and cancerous regions within the tissue sections. Applying a 'support vector machine' algorithm to classify the cancerous from the noncancerous regions, an overall cross-validation, a sensitivity and specificity of $88,85.21$ and $90.74 \%$, respectively, was achieved. Additionally four distinctively overexpressed peaks were identified: 2,753 and 6,704 Da for non-cancerous glands, and 4,964 and 5,002 Da for cancerous glands. The results of this first clinical study utilizing the new technique of MALDI-Imaging underline its vast potential to identify candidates for more reliable prostate cancer tumor markers and to enlighten the pathogenesis of prostate cancer.
\end{abstract}

\section{Introduction}

Prostate cancer $(\mathrm{PCa})$ is one of the most common malignancies in men worldwide including Germany. Estimated numbers of

Correspondence to: Professor Axel Wellmann, Institute of Pathology, RWTH Aachen University, Pauwelsstr. 30, 52074 Aachen, Germany

E-mail: awellmann@ukaachen.de

${ }^{*}$ Contributed equally

Key words: Matrix-assisted laser desorption/ionization-Imaging, prostate cancer, proteomics, pattern recognition, profiling the German Cancer Society for 2002 included 48,650 $(22.3 \%)$ prostate cancer diagnoses and 11,422 (10.4\%) prostate cancer deaths (1). Detecting PCa is based on serum measurement of prostate-specific antigen (PSA) together with a digital rectal examination (2). Both diagnostic tools (alone or in combination) however lack specificity (3-6). Especially, PSA values $<15 \mu \mathrm{g} / 1$ cannot distinguish between carcinoma and benign prostate hyperplasia (BPH) (7). Above all, elevated PSA levels can be caused by different diseases apart from cancer, e.g. prostatitis and BPH, respectively (8). Excluding these external factors, variations in PSA levels may even range as much as $\pm 30 \%$ over a three-month observation period (9) resulting in a positive predictive value of only $47 \%$ (10). According to the current literature there is a consensus, that at the present time only the size of the prostate and not the size of the actual carcinoma is represented by the PSA value (11).

Additionally, a currently unsolved problem is the determination of the clinical significance of the diagnosed $\mathrm{PCa}$. For example, the risk that early-stage PCa will develop into significant PCa is believed to be quite low (12) and is probably only life-threatening in up to $10 \%$ of cases (13). However, due to the absence of a reliable and accurate biomarker to identify significant PCas and to monitor nonsignificant $\mathrm{PCas}$, all patients are treated as harboring significant PCa.

Proteomics is a versatile new science. Its usage ranges from basic research to clinical proteomics (14). The latter focuses on potential diagnostic application. Expression protein profiling methods provide promising results regarding the identification of biomarkers capable of distinguishing between normal and disease states, which would especially benefit prostate cancer diagnosis (15). Typically, cells of interest within tissue samples must be microdissected carefully and lysed prior to matrix-assisted laser desorption/ ionization time of flight (MALDI-TOF) mass spectrometry (MS) (16). The techniques used are well established but tedious (17). In addition the minimal requirement of protein content (cell number) may be difficult to obtain. One emerging and promising new technique for protein analysis from intact biological tissues is MALDI-Imaging mass spectrometry (IMS) (18). Basically it is a development from the wellestablished single-cell detection techniques based on MALDI-TOF $(19,20)$. A single cryostat section is sufficient for analysis. Therefore this method overcomes all of the 
problems described above and additionally enables access to the spatial distribution of proteins in a tissue sample (21). Moreover, this information can be correlated with histologic features of the same section by optical microscopy $(22,23)$.

In this report we present the results of an initial clinical study using IMS on cancerous and non-cancerous prostate sections. We discovered proteomic alterations, which may facilitate the understanding of this highly variable disease and could lead to the identification of potential new marker molecules for prostate cancer. Furthermore we proved the feasibility of this new technique resulting in a good spatial distribution of protein even in a complex heterogeneous tissue section.

\section{Materials and methods}

Sample collection and patient specimens. Tissue specimens were collected from patients undergoing a radical prostatectomy at the Department of Urology, RWTH Aachen University, under routine protocol. Briefly, tissue from the peripheral zone at the dorsal part of the prostate was obtained from fresh prostatectomy specimens, immediately snap frozen and stored at $-80^{\circ} \mathrm{C}$. One cryosection was $\mathrm{H} \& \mathrm{E}$ stained for examination by a board-certified pathologist for histological classification. Eleven samples with cancer and 11 samples without cancer were used for this study. Gleason score was 6 (4), 7 (4) and 9 (3), respectively. All specimens were procured from patients giving informed consent and with the approval of the Ethics Commission (EK 122/04).

Sample preparation. Samples were frozen on a cryostat steel plate using as little OCT (Optimal Cutting Temperature) polymer as possible to avoid contamination of the section with OCT. Sections (10 $\mu \mathrm{m}$ thick) were cut with a stainless steel microtome blade and mounted onto conductive glass slides (Bruker Daltonics, Germany). Slides were washed in $70 \%$ ethanol (HPLC grade) for $30 \mathrm{sec}$ and in $96 \%$ ethanol (HPLC grade) for $15 \mathrm{sec}$. Sections were allowed to dry and were stored at $-80^{\circ} \mathrm{C}$. Prior to matrix application, slides were marked with dots of liquid white-out, and native sections were photographed using a low magnification microscope. Matrix solution consisted of $180 \mathrm{mg}$ sinapinic acid matrix (Bruker, \#203073) in 5,000 $\mu 1$ LC-MS water, 5,000 $\mu 1$ acetonitrile and $10 \mu 1$ TFA (tri-fluoro acetic acid) (all high pure reagents from Sigma). Sections were carefully spray coated using a TLC (Thin Layer Chromatography) spraying device (Sigma) made of glass. Repeated spray-drying cycles were performed at a $30 \mathrm{~cm}$ distance from the slide to obtain an optimal matrix deposition. Care was taken to standardize the matrix application throughout the case number.

Following the imaging experiments as described below, slides were incubated twice in methanol $(100 \%$, at room temperature for $5 \mathrm{~min}$ ) and once in acetone $(100 \%$, at room temperature for $5 \mathrm{~min}$ ) to remove the matrix and optimize tissue fixation. After air drying, slides were stained using regular H\&E protocol (24) to confirm diagnosis by a boardcertified pathologist and to correlate MS data with the histological features of the same section.
MS analysis. All imaging experiments were performed on a Reflex IV MALDI-TOF-MS (Bruker) using FlexImaging 2.0 software (Bruker) to control the analysis. The picture of the native section together with the liquid white-out dots was used to teach the software. At each measuring point 20 sufficient laser shots were averaged per spectrum. The spot raster was set to $200 \mu \mathrm{m}$ resulting in a good compromise between the resulting file size and spatial resolution power.

Data analysis was performed using ClinProTools 2.0 software (Bruker) to distinguish between normal and cancerous tissue. Therefore, regions of interest were designated on each sample representing normal and cancerous areas, respectively. The latter consisted typically of about 50 single measuring points. The spectra of these regions were exported to ClinProTools and statistical analysis was performed using a five-dimensional genetic algorithm and a 'support vector machine' algorithm (SVM), respectively. According to the Bruker homepage www.bdal.de, the genetic algorithm is a stochastic search algorithm, which mimics evolution in nature. It is used for the optimization of an objective function (fitness function) for a large number of solutions, which are termed peak combinations. It considers many possible peak combinations simultaneously. To this the genetic algorithm defines numerous preselections of five peaks, which are tested for their classifying capability. The SVM is historically a classifier and not a feature selection algorithm. SVM tries to find a hyperplane that separates one or more classes. In the simplest case, the SVM helps to determine an optimal hyperplane separating two clouds of data. The algorithm tries to find this line in a multidimensional space. Our generated models were then used to classify each spectrum of the sample into the normal or cancerous group. Exporting these data into FlexImaging enabled us to visualize the results of the section classification in a color-encoded depiction.

\section{Results}

Using MALDI-Imaging mass spectrometry on prostate tissue sections resolved on average up to 85 peaks ranging from 1-20 $\mathrm{kDa}$. On every section, the regions of interest were defined, resembling representative regions of predominantly cancerous and non-cancerous glands, respectively. Fig. 1 illustrates the overall sum spectra of these regions of interest, showing protein profiles from 1-15 $\mathrm{kDa}$. Within this mass range a number of differentially expressed proteins were detected. After exporting the spectra obtained within these regions to ClinProTools software, we were able to identify a proteomic pattern, which in turn clearly distinguished between cancerous and non-cancerous regions within the prostate tissue section. Based on an SVM algorithm generated by the software, cancerous and non-cancerous regions were discriminated with an overall cross-validation of $88 \%$ resulting in a sensitivity and a specificity of 85.21 and $90.74 \%$, respectively. This algorithm was based on 22 peaks of different masses ranging from 1.4-12.4 kDa. Four out of these 22 peaks were of special interest due to their higher impact on the classification: 2,753 and 6,704 Da for noncancerous glands, and 4,964 and 5,002 Da for cancerous glands, respectively. Secondarily a five-dimensional genetic algorithm was generated, resulting in an overall cross- 


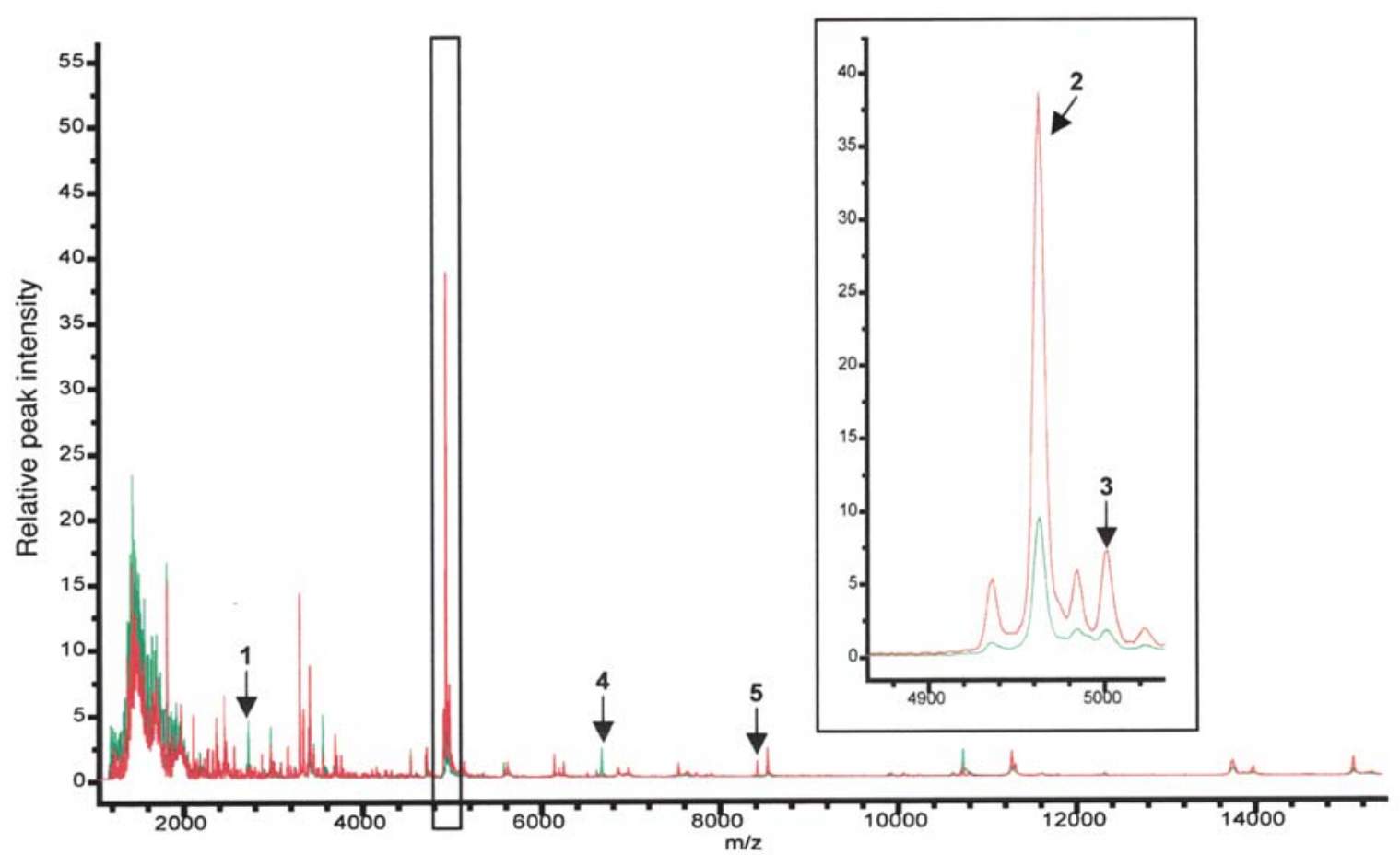

Figure 1. Overall sum spectra in the mass range of 1-15 kDa (m/z) obtained from all cancerous regions of interest (red) and all non-cancerous regions of interest (green) from all 22 sections, respectively. Arrows indicate the five peaks used to generate the genetic algorithm.
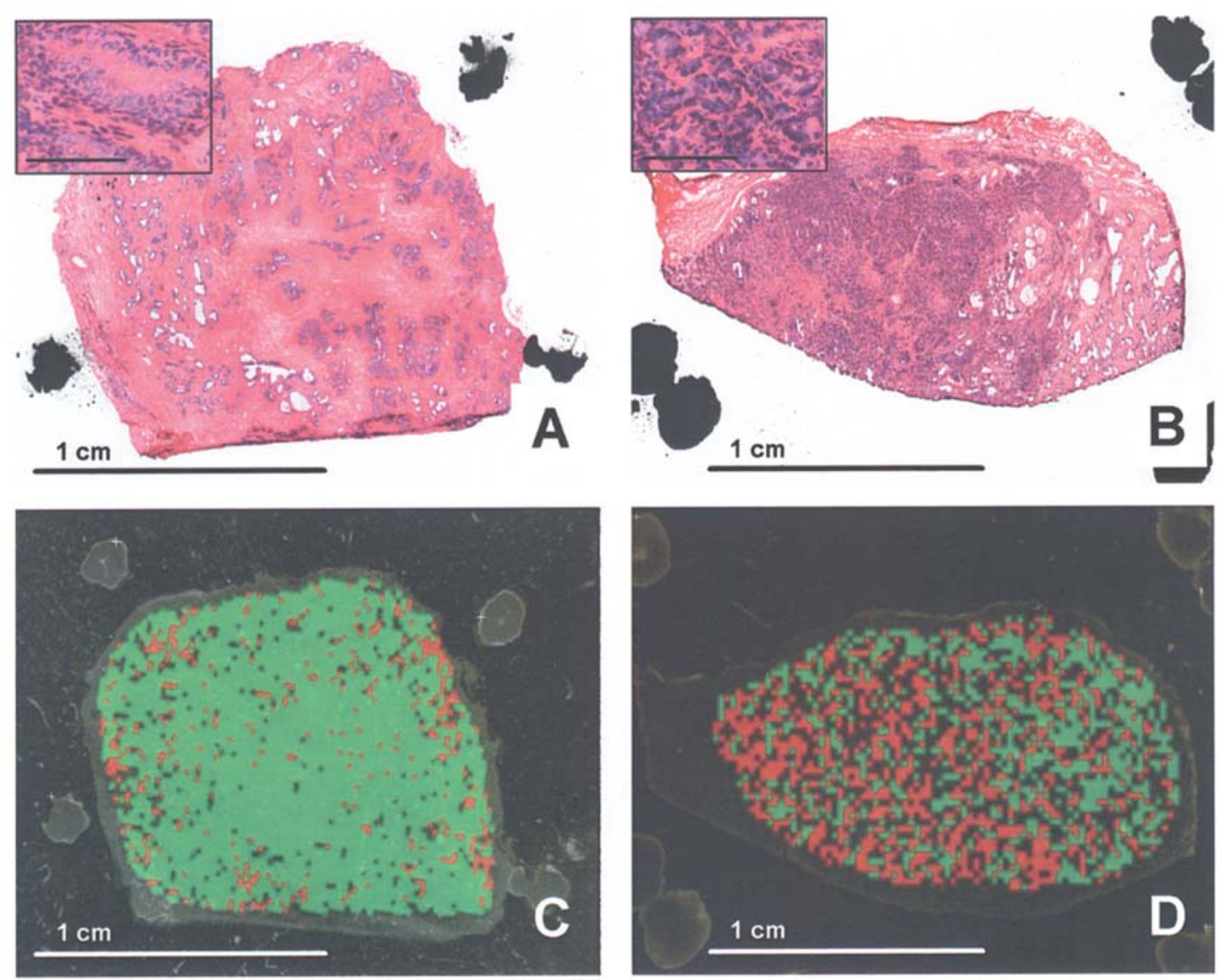

Figure 2. Representative images of H\&E-stained sections of normal prostate tissue (A) and prostate tissue with cancerous regions (B). The inserts show enlarged views of the regions of interest with non-cancerous glands (A) and cancerous glands (B) (bar, $10 \mu \mathrm{m}$ ). Class images of the very same sections: normal prostate tissue (C) and prostate tissue with cancerous regions (D). Green, normal; red, cancerous, and non-colored, unclassified. H\&E staining was performed after mass spectrometric analysis and removal of the matrix. Black points (A and B) and grey points (C and D) visible near tissue sections resemble teaching points with liquid white-out. 
validation, sensitivity and specificity of 77,70 and $84 \%$, respectively. The five peaks used in the algorithm are marked in Fig. 1. Their masses ranged from 2.7-8.5 kDa and contained the four masses from the SVM algorithm mentioned above.

When spectra obtained by IMS from the sections were imported into ClinProTools software, classification of each spectrum into one of the two different classes, cancerous and normal, was possible. Therefore the generated support vector machine algorithm was applied. Two representative sections, one without and one with cancer are depicted in Fig. 2. H\&Estained sections (Fig. 1A and B) were the same sections used for imaging. Staining was performed after imaging and removal of the matrix. By exporting the classification results back into the FlexImaging software, we generated a class image (Fig. 2C and D). Green was used for non-cancerous tissue and red for cancerous tissue. Pixels neither green nor red were due to unclassified spectra. The class image resembled the H\&E image making cancerous regions easily visible.

\section{Discussion}

The ongoing search for new and reliable tumor markers for prostate cancer is underlined by the fact that it has become one of the most commonly diagnosed malignancies in men worldwide (25). Identifying such biomarkers could improve tumor diagnosis and treatment as well as cost efficient management. The latter is of growing importance in light of shrinking health care budgets. Both molecular and proteomic studies every so often have been hampered by the limited availability of the cells of interest, which need to be isolated from heterogeneous tissue by tedious microdissection techniques. The number of lysed cells necessary for a single MS spectrum ranges from 1,000 (26) to 50,000 (27) depending on the sensitivity necessary and the protocol used. IMS was initially developed out of single-cell detection techniques $(19,20)$, which in turn do not need cellular lysates but analyze single intact cells. The next logical step towards a 2-D layer of cells (tissue section) was subsequently performed by Caprioli and coworkers (28), who basically invented the method of IMS and developed most of the contemporary protocol. IMS allows for the correlation of MS-driven data with histologic images avoiding microdissection by an elegant manner. It overcomes most problems associated with the limitation of biological sample material, as it requires only one frozen tissue section for highly sensitive proteomic analysis resulting in a proteome map. The latter contains high spatial resolution and allows the correlation of this information with histomorphologic features of the very same tissue section.

By comparing cancerous and non-cancerous regions we identified several proteins upregulated and downregulated respectively in a class-specific pattern. Using a 'support vector machine' algorithm, both region types could be clearly distinguished from each other with an overall cross-validation, a sensitivity and specificity of 88,85.21 and 90.74\%, respectively. By applying this algorithm to every spectrum of a section obtained by IMS, a class image was generated. Therein, spectra classified into the cancerous group were represented by red pixels and spectra out of the non-cancerous group by green pixels. When comparing the class image with
H\&E images of the very same section, cancerous and noncancerous regions on both images were congruent and easily visible. At first sight this new technique seemed to only confirm a histological-driven diagnosis of malignancies with information of protein expression. With a protein signature however, which is disease specific, a diagnostic tool was created. Therefore the next logical step will be address the identification of differentially expressed proteins identified in this study.

Unclassified spectra, represented by non-colored pixels were due to either spectra that neither belonged to the cancerous nor to the non-cancerous group or spectra of nonsufficient quality, i.e. null spectra. The latter is a result of the process of matrix application. By spray coating with a TLC sprayer, even when performed in a standardized manner and with great care, some areas of the sections were covered with less matrix than other areas (data not shown). This problem can be overcome by applying the matrix in an automated manner by machine.

As this is the first study applying this new technique on prostate tissue sections, the only studies with which to compare are based on tissue lysates. Cazares and coworkers (29) identified several small molecular mass peptides or proteins differentially expressed in normal, benign, preneoplastic and malignant epithelial cells utilizing surfaceenhanced laser desorption/ionization (SELDI) mass spectrometry. In $22 \%$ of the preneoplastic and $29 \%$ of the malignant epithelial cells, an overexpression of an 8,445 Da protein was found when compared with matched normal samples. Additionally two other proteins (3,448 and 4,749 Da) were overexpressed in cancerous samples and a 5,666 Da protein was mostly overexpressed in BPH samples. In our study we detected a 3,441 and an 8,450 Da protein with increased expression in cancerous regions of the prostate. Two other proteins $(4,747$ and 5,652 Da) showed a slight overexpression in the cancerous regions. Minor differences in the masses may have been due to the differences in the two approaches that were utilized.

In another study Wright and coworkers (30) described a 3,574 Da protein upregulated in PCa samples when compared with normal prostate cell lysates using SELDI. When applying IMS we identified a 3,588 Da protein overexpressed in normal regions. The differences in the protein mass and the expression status may have been the result of applying different techniques.

Several other MS-based studies were able to pinpoint different proteins upregulated and downregulated in cancerous prostate gland and the adjacent stroma (31-33) that we were not able to confirm.

In this study we were able to demonstrate the feasibility of IMS for classification of prostate tissue samples. We identified differential protein expression between normal and malignant regions of the prostate sections. Using pattern recognition algorithms a sensitive and specific classification model was generated. IMS is a promising technique for proteomic maps of single cryostat sections with high spatial resolution. In addition MS data can be correlated to histomorphological information. IMS analysis has potential clinical importance as it may support or enable computerassisted clinical evaluation of tissue specimens. 


\section{Acknowledgements}

The authors thank Peggy Jirak, Nadine Reulen and Sandra Uhlig for the technical assistance and Dr Sören Deininger, Bruker Daltonik GmbH, Bremen, Germany, for his help and advice.

\section{References}

1. Gesellschaft der epidemiologischen Krebsregister in Deutschland. Krebs in Deutschland. Häufigkeiten und Trends. 5. ueberarbeitete Auflage, 2006.

2. Aus G, Abbou CC, Bolla M, Heidenreich A, Schmid HP, Van Poppel H, Wolff J and Zattoni F: European Association of Urology. EAU guidelines on prostate cancer. Eur Urol 48: 546-551, 2005.

3. Hsu CY, Joniau S, Oyen R, Roskams T and Van Poppel H: Detection of clinical unilateral T3a prostate cancer - by digital rectal examination or transrectal ultrasonography? BJU Int 98: 982-985, 2006.

4. Lein M, Semjonow A, Graefen M, et al: A multicenter clinical trial on the use of $(-5,-7)$ pro prostate-specific antigen. J Urol 174: 2150-2153, 2005 .

5. Brawer MK: Prostate-specific antigen: current status. CA Cancer J Clin 49: 264-282, 1999.

6. Stenman U-H, Leinonen J, Zhang W-M and Finne P: Prostatespecific antigen. Semin Cancer Biol 9: 83-93, 1999.

7. Peter J, Unverzagt $\mathrm{C}$ and Hoesel W: Analysis of free prostatespecific antigen (PSA) after chemical release from the complex with alpha(1)-antichymotrypsin (PSA-ACT). Clin Chem 46: 474-482, 2000.

8. Nadler RB, Humphrey PA, Smith DS, Catalona WJ and Ratliff TL: Effect of inflammation and benign prostatic hyperplasia on elevated serum prostate specific antigen levels. J Urol 154: 407-413, 1995

9. Bangma $\mathrm{CH}$ and Verhagen PC: Blood and serum substances for markers of prostate cancer. Microsc Res Tech 51: 430-435, 2000.

10. Brawer MK, Benson MC, Bostwick DG, Diavan B, Lilja H, Semjonov A, Su S and Zhou Z: Prostate-specific antigen and other serum markers: Current concepts from the World Health Organization Second International Consultation on prostate cancer. Semin Urol Oncol 17: 206-221, 1999.

11. Stamey TA, Caldwell M, McNeal JE, Nolley R, Hemenez M and Downs J: The prostate specific antigen era in the United States is over for prostate cancer: What happened in the last 20 years? J Urol 172: 1297-1301, 2004.

12. Etzioni R, Penson DF, Legler JM, di Tommaso D, Boer R, Gann PH and Feuer EJ: Overdiagnosis due to prostate-specific antigen screening: Lessons from U.S. prostate cancer incidence trends. J Natl Cancer Inst 94: 981-990, 2002.

13. Coffey DS: Prostate cancer. An overview of an increasing dilemma. Cancer 71 (suppl 3): 880-886, 1993.

14. Krieg RC, Paweletz CP, Liotta LA and Petricoin EF III: Clinical proteomics for cancer biomarker discovery and therapeutic targeting. Technol Cancer Res Treat 1: 263-272, 2002.

15. Wright ME, Han DK and Aebersold R: Mass spectrometrybased expression profiling of clinical prostate cancer. Mol Cell Proteomics 4: 545-554, 2005.
16. Hillenkamp F and Karas M: Mass spectrometry of peptides and proteins by matrix-assisted ultraviolet laser desorption/ ionization. Methods Enzymol 193: 280-295, 1990.

17. Krieg RC, Gaisa NT, Paweletz CP and Knuechel R: Proteomic analysis of human bladder tissue using SELDI-approach following microdissection-techniques. Methods Mol Biol 293: 255-267, 2005

18. Chaurand P, Stoeckli M and Caprioli RM: Direct profiling of proteins in biological tissue sections by MALDI mass spectrometry. Anal Chem 71: 5263-5270, 1999.

19. van Veelen PA, Jiménez CR, Li KW, Wildering WC, Geraerts WPM, Tjaden UR and van der Greef J: Direct peptide profiling of single neurons by matrix-assisted laser desorptionionization mass spectrometry. Org Mass Spectrom 28: 1542-1546, 1993

20. Claydon MA, Davey SN, Edwards-Jones V and Gordon DB The rapid identification of intact microorganisms using mass spectrometry. Nat Biotechnol 14: 1584-1586, 1996.

21. Caldwell RL and Caprioli RM: Tissue profiling by mass spectrometry. Mol Cell Proteomics 4: 394-401, 2005

22. Chaurand P, Schwartz SA, Billheimer D, Xu BJ, Crecelius A and Caprioli RM: Integrating histology and imaging mas spectrometry. Anal Chem 76: 1145-1155, 2004.

23. Crecelius AC, Cornett D, Caprioli RM, Williams B, Dawant BM and Bodenheimer B: Three-dimensional visualization of protein expression in mouse brain structures using imaging mass spectrometry. J Am Soc Mass Spectrom 16: 1093-1099, 2005.

24. Burck HC: Histologische Technik. Georg Thieme Verlag, Stuttgart, Germany, 1988

25. Lee SW, Lee KI and Kim JY: Revealing urologic diseases by proteomic techniques. J Chromatogr B Analyt Technol Biomed Life Sci 815: 203-213, 2005.

26. Krieg RC, Fogt F, Braunschweig T, Herrmann PC, Wollscheidt V and Wellmann A: ProteinChip array analysis of microdissected colorectal carcinoma and associated tumor stroma shows specific protein bands in the 3.4 to $3.6 \mathrm{kDa}$ range. Anticancer Res 24: 1791-1796, 2004

27. Zheng Y, Xu Y, Ye B, et al: Prostate carcinoma tissue proteomics for biomarker discovery. Cancer 98: 2576-2582, 2003.

28. Caprioli RM, Farmer TB and Gile J: Molecular imaging of biological samples: localization of peptides and proteins using MALDI-TOF-MS. Anal Chem 69: 4751-4760, 1997.

29. Cazares LH, Adam BL, Ward MD, Nasim S, Schellhammer PF, Semmes OJ and Wright GL: Normal, benign, preneoplastic, and malignant prostate cells have distinctive protein expression profiles revealed by surface enhanced laser desorption/ ionization mass spectrometry. Clin Cancer Res 8: 2541-2552, 2002.

30. Wright GL Jr, Cazares LH, Leung SM, et al: Proteinchip ${ }^{\circledR}$ surface enhanced laser desorption/ionization (SELDI) mass spectrometry: a novel protein biochip technology for detection of prostate cancer biomarkers in complex protein mixtures. Prostate Cancer Prostatic Dis 2: 264-276, 1999.

31. Wellmann A, Wollscheid V, Lu H, et al: Analysis of microdissected prostate tissue with ProteinChip ${ }^{\circledR}$ arrays - a way to new insights into carcinogenesis and to diagnostic tools. Int J Mol Med 9: 341-347, 2002 .

32. Cheung PK, Woolcock B, Adomat $\mathrm{H}$, et al: Protein profiling of microdissected prostate tissue links growth differentiation factor 15 to prostate carcinogenesis. Cancer Res 64: 5929-5933, 2004.

33. Liu AY, Zhang H, Sorensen CM and Diamond DL: Analysis of prostate cancer by proteomics using tissue specimens. J Urol 173: 73-78, 2005 . 